\title{
Optical spectra of five UX Orionis-type stars ${ }^{\star, \star \star}$
}

\author{
V. P. Grinin ${ }^{1,2}$, O. V. Kozlova ${ }^{1}$, A. Natta ${ }^{3}$, I. Ilyin ${ }^{4}$, I. Tuominen ${ }^{4}$, \\ A. N. Rostopchina ${ }^{1}$, and D. N. Shakhovskoy ${ }^{1}$
}

\author{
1 Crimean Astrophysical Observatory, Crimea, 334413 Nauchny, Ukraine \\ 2 Pulkovo Astronomical Observatory, 196140 St. Petersburgh, Russia \\ 3 Osservatorio Astrofisico di Arcetri, Largo E. Fermi 5, 50125 Firenze, Italy \\ 4 Astronomy Division, PO Box 3000, 90014, University of Oulu, Finland
}

Received 28 Mars 2001 / Accepted 7 September 2001

\begin{abstract}
We present in this paper several high resolution $(R=27000)$ spectra of five UXORs (UX Ori, CQ Tau, BF Ori, RR Tau, WW Vul), which cover the entire visual range, from 3900 to about $8700 \AA$. There are between 4 and 7 spectra per star, obtained over a time interval of two years. Simultaneous or quasi-simultaneous photometric observations were also obtained at the Crimean Astrophysical Observatory. The complete, reduced and normalized spectra are available in electronic form. We show for each star a selection of the most interesting lines, and the full spectrum of UX Ori computed by averaging the spectra obtained when the star was at maximum light. For UX Ori we show also the synthetic spectrum and provide an identification of most of the lines. The spectra are too sparse to form true time sequences; however, they provide an extremely useful database for studies of UX Ori-type stars. We discuss briefly the main features of the spectra. We show that they contain many time-stable photospheric lines that can be described to a good approximation by the synthetic spectra of normal A stars with $\log g=3.5-4$ and we derive for each star effective temperature, gravity and rotational velocity. We examine the time variability of selected lines and study their connection with the photometric activity of the stars. Two different types of spectral variability are identified. One is common to all stars with circumstellar (CS) gas and is caused by perturbations of the physical and kinematic conditions of the emitting region. There is no correlation between this type of activity and the brightness variations of the star. On the contrary, a second type of spectral variability correlates well with the brightness variations and is very likely connected with the screening effect of an opaque dust cloud which sporadically intersects the line of sight. This type of variability has been observed in its simplest form in one of the RR Tau spectra, where the equivalent width of the forbidden line [O I] $6364 \AA$ increased by a factor of about three as the star faded by approximately the same amount.
\end{abstract}

Key words. stars: individual: UX Ori, CQ Tau, BF Ori, RR Tau, WW Vul - stars: general

\section{Introduction}

This paper presents the results of high resolution spectroscopic observations of five pre-main-sequence stars of intermediate mass belonging to the UXORs group. UXORs are pre-main-sequence stars of intermediate mass, mostly found among Herbig Ae stars (HAe in the following), characterized by their typical photometric and polarimetric variability (see reviews by Thé 1994; Grinin 1994 and references therein). They all show sporadic deep minima at visual wavelengths (typically of $2-3 \mathrm{mag}$ ), during which the fraction of polarized light increases from typical

\footnotetext{
Send offprint requests to: A. Natta,

e-mail: natta@arcetri.astro.it

* Based on observations made with the Nordic Optical Telescope, operated on the island of La Palma jointly by Denmark, Finland, Iceland, Norway, and Sweden, in the Spanish Observatorio Roque de los Muchachos of the Instituto de Astrofisica de Canarias.

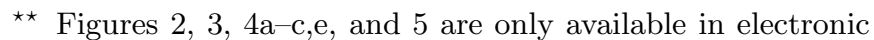
form at http://www.edpsciences.org
}

interstellar values to a few percent. As the star fades, the radiation first becomes redder, then bluer again. The best interpretation of these phenomena requires that the star is surrounded by a disk, which scatters a fraction of the stellar radiation toward the observer (Grinin 1988). The minima are caused by a much larger than average column of intervening dust, which occasionally occults the star. At that point, the fraction of stellar radiation received directly by the observer drops, while the fraction seen after being scattered by the disk remains practically unchanged and represents a significant contribution to the total. It is likely that variable extinction is present in most HAe stars, but that we see its effects only when the circumstellar disks are seen under small inclination angles to the line of sight (Grinin et al. 1991; Natta \& Whitney 2000).

The spectra of UXORs show a number of lines of photospheric and circumstellar origin. Several of them (D NaI, He I 5876, H $\alpha$ are highly variable (Graham 1992; Grinin et al. 1994; Grady et al. 1996; de Winter 1999; Kozlova et al. 2000), showing evidence for complex 
motions of the circumstellar gas in the immediate vicinity of the star. Red-shifted absorption components are frequently seen in the $\mathrm{Na}$ I resonance lines and in some other metallic lines, as well as in the hydrogen Balmer lines. These components seem to appear and disappear on timescales of days or shorter, indicating infall of gas clumps onto the star, similar, apparently, to infalling events observed in the spectrum of $\beta$ Pictoris. The origin of such accretion events in UXORs is matter of debate (Sorelli et al. 1996; Grinin et al. 1996; Pérez \& Grady 1997; de Winter et al. 1998; Grady et al. 2000; Natta et al. 2000a).

It is clear from all previous works that the understanding of the spectral variability of UXORs requires spectra which include many lines of different species over a wide range of wavelengths. Moreover, it is important to have simultaneous information on the brightness of the star, since the comparison of spectra taken when the star is in a deep minimum to those obtained at maximum light provides invaluable clues to the physical processes which take place in the circumstellar environment.

Many UXORs (including UX Ori itself) are among Herbig Ae/Be stars which are not associated with bright nebulosities, so that most of them were not included either in the original list of Herbig Ae/Be stars (Herbig 1960), in those of Finkenzeller \& Mundt (1984) and Hamann \& Persson (1992), but only in the most recent catalogue of Thé et al. (1994). This is the main reason why detailed spectroscopic studies of UXORs have begun to appear only recently (see, for example, de Winter et al. 1999; Eiroa et al. 2000).

The main purpose of this paper is to make available to the community a number of high resolution spectra of 5 of the photometrically most active stars of this type: UX Ori, CQ Tau, WW Vul, RR Tau, and BF Ori. For each star, we have between 4 and 7 spectra taken over a time interval of about two years. Each spectrum covers, with some exceptions, the whole optical region from 3900 to about $8700 \AA$. The amount of information they contain is huge. Although some of it has already been used by us in studies of UXORs properties (Natta et al. 2000b; Tambovtseva et al. 2001), and more work will follow, we think that the spectra may be of value for other groups as well.

Observations and data reduction are described in Sect. 2. We provide in Sects. 3 and 4 a brief discussion of the main features observed in the spectra. In Sect. 5 we re-determine the stellar parameters by comparing the most stable absorption features to the prediction of synthetic spectra. Some implications are briefly discussed in Sect. 6. The properties of the stars, as known from the literature, are summarized in Table 1.

\section{Observations and data reduction}

High-resolution spectra were collected with the SOFIN échelle spectrograph (Tuominen et al. 1999) at the $2.56 \mathrm{~m}$ Nordic Optical Telescope (NOT) during two observing runs in 1995 and 1996 (we thank P. Petrov for giving us time to obtain the first assessment spectrum in 1994).

We used the 3rd camera, which provides a spectral resolution per $2 \mathrm{CCD}$ pixels of about $12 \mathrm{~km} \mathrm{~s}^{-1}$ with the entrance slit width of $1^{\prime \prime} 7(R=27000)$. The EEV P88200 $1152 \times 770$ pixels CCD was used as a detector, whose format allows coverage of all 43 spectral orders in one exposure. Given the length of each spectral order (about $140 \AA$ around $\mathrm{H} \alpha$ ), two different spectral settings are necessary to cover the whole spectrum from 3700 to $10700 \AA$ in two exposures.

The CCD images of the échelle spectra were obtained and reduced with the $4 \mathrm{~A}$ software package (Ilyin 2000). The standard procedure involves bias subtraction, correction for the master flat field, scattered light subtraction with aid of 2D-smoothing splines, definition of spectral orders, and weighted integration of the intensity along with elimination of cosmic spikes.

The wavelength calibration was done with a ThAr comparison spectrum. To reduce the effect of line drifts during long integrations caused by temporal changes in a Cassegrain mounted spectrograph, the long exposures were subdivided into $2-3$ shorter ones with the ThAr spectrum taken at the beginning of the series. For each image, we used the Gaussian centered positions and wavelengths of all detected ThAr lines from all spectral orders, obtaining an accuracy of the global fit of about $50-80 \mathrm{~ms}^{-1}$ in the image center.

Temporal variations in the spectrograph during integration due to change of its orientation, ambient temperature, CCD dewar nitrogen evaporation, etc., as well as the slit effect caused by variations of the collimator illumination due to change of the seeing and the differences in the placement of the star on the slit from one observation to another, all result in a small but significant spurious shift of the stellar spectral lines which cannot be completely eliminated with the ThAr spectrum. With the aid of the telluric lines, numerous in the red orders, the wavelength scale was corrected for the spurious shift, where the offset was determined by cross-correlation of the telluric orders with respect to an artificial telluric spectrum (see Ilyin \& Dümmler 1999 for details). Finally, the wavelength scale of the spectra was corrected for the orbital and diurnal motions of the Earth, and the radial velocity of the star.

The bell-like shape of the stellar spectra caused by the vignetting in the 3rd short focus optical camera was corrected with use of the flat field échelle spectra taken after each object exposure. Although the shape of the vignetting function in the stellar spectrum and flat field are alike, a small discrepancy arise from the difference in the optical path of the seeing image and flat field source. Therefore, the correction for the vignetting function corrects the continuum, but may introduce some large scale deviations which are then eliminated with a continuum fitting.

We have removed the numerous telluric $\mathrm{H}_{2} \mathrm{O}$ lines that contaminate the spectra especially in the red using the spectrum of the reference stars $\beta$ Eri (A3III) and 59 Tau 
Table 1. Stellar parameters.

\begin{tabular}{lccccccc}
\hline \hline Name & Sp & $\begin{array}{c}D \\
(\mathrm{pc})\end{array}$ & $\begin{array}{c}L \\
\left(L_{\odot}\right)\end{array}$ & $\begin{array}{c}\text { Age } \\
(\mathrm{Myr})\end{array}$ & $\begin{array}{c}\Delta V \\
(\mathrm{mag})\end{array}$ & $\begin{array}{c}\text { Max. Pol. } \\
(\%)\end{array}$ & $\begin{array}{c}v \sin i \\
\left(\mathrm{~km} \mathrm{~s}^{-1}\right)\end{array}$ \\
\hline UX Ori & A3III & 430 & 39 & 4 & 3.9 & 6.7 & 70 \\
CQ Tau & F2IV & 100 & 6.6 & 10 & 2.2 & 7.9 & 110 \\
BF Ori & A5III & 460 & 39 & 2.3 & 3.0 & 5.7 & 100 \\
RR Tau & A0III-IV & 380 & 37 & 3 & 3.7 & 6.8 & - \\
WW Vul & A3III & 550 & 29 & 4 & 2.2 & 5.3 & - \\
\hline \hline
\end{tabular}

Comments: Spectral types and luminosities are taken from Rostopchina (1999), the distances and ages for UX Ori and CQ Tau from Natta et al. (1997), for the other stars from Rostopchina (1999). The maximum amplitude $\Delta \mathrm{V}$ and linear polarization of minima are from the Crimean data base. The values of $v \sin i$ from Böhm \& Catala (1993).

(B9V) observed during the same nights with about the same zenith angles. We used also for this purpose the telluric lines of the solar spectrum (Wallace et al. 1998). In this case the water lines were broadened by the instrumental profile and normalized to the same air mass.

The spectra are not flux-calibrated. We have normalized each spectral order to the observed continuum. This is generally easy, with the exception of those wavelength intervals dominated by the broad photospheric wings of the Balmer lines. In these cases we have used the synthetic spectra (see Sect. 5) for control.

A logbook of the observations is given in Table 2, which lists in Col. 1 the name of the star, in Col. 2 the epoch of the observation, in Col. 3 an estimate of the $V$ magnitude of the star at that epoch, in Col. 4 the spectral setting (1 or 2 ) used in the observations, in Col. 5 the exposure time, in Col. 6 the signal-to-noise at $\lambda=6400 \AA$, and in Col. 7 the equivalent width of $\mathrm{H} \alpha$ (see Sect. 3.1). Note that $S / N$ decreases toward the blue part of the spectrum and at the end of each order, so that the quality of the data may vary from region to region.

The NOT spectral observations were accompanied by photometric observations with the Crimean Observatory $1.25 \mathrm{~m}$ telescope equipped with a five-channel photometerpolarimeter (Piirola 1975). The procedure and data reduction of these observations have been described in Grinin et al. (1988). The stellar magnitudes of the program stars in the $V$ band are given in Table 2. In the majority of cases the photometric observations were carried out on the same night as the spectroscopic ones, in some cases the night before or after. In a few cases the time interval between the spectral and photometric observations was more then one day, and they are marked by a semicolumn in Table 2. Three stars showed significant photometric variability during the nights covered by the spectral observations, although no spectrum has been obtained for stars in deep minima. They are BF Ori $(\Delta V=0.98)$, $\mathrm{CQ} \operatorname{Tau}(\Delta V=0.80)$ and $\mathrm{RR} \operatorname{Tau}(\Delta V \approx 1.6)$. All the spectra of UX Ori and WW Vul were obtained when the stars were close to their bright state.

\section{General description of the spectra}

The spectra are available in ascii form (wavelength and normalized fluxes for each of the nights) upon request ${ }^{1}$.

To illustrate the potential of these (and similar) observations, we have produced a first group of figures which show for each star a selection of the most interesting circumstellar lines that display variability. For each line we plot the normalized profile as function of velocity shift at different epochs. For several of the lines we also plot the synthetic photospheric spectrum (see Sect. 5). The figures show the results for UX Ori (Figs. 1a-e), CQ Tau (Figs. 2a-e), BF Ori (Figs. 3a-e), RR Tau (Figs. 4a-e) and WW Vul (Figs. 5a-d). Only Fig. 1 (all the variable lines in the spectrum of UX Ori) and Fig. $4 \mathrm{~d}$ (displaying the [OI] $6363 \AA$ at various epochs in $\mathrm{RR}$ Tau) are shown in print, the others are available only electronically; nevertheless, we will refer to them in the following sections of this paper.

In Fig. 6 we show the complete spectrum of UX Ori, computed by averaging the spectra of the three 1996 nights when the star was at maximum brightness. The full spectrum includes 27 panels ordered from "a" to "zz" in order of increasing wavelength. We show in print two selected wavelength spectral intervals; the rest of the figures is available electronically (in colors). Figure 6 shows also the complete synthetic spectrum $\left(T_{\text {eff }}=9500 \mathrm{~K}\right.$ and $\log g=4.0)$, before and after convolution with the projected rotation velocity $\left(v \sin i=140 \mathrm{~km} \mathrm{~s}^{-1}\right)$. Most of the

\footnotetext{
1 Contact grinin@VG1723.spb.edu.
} 
UX Ori
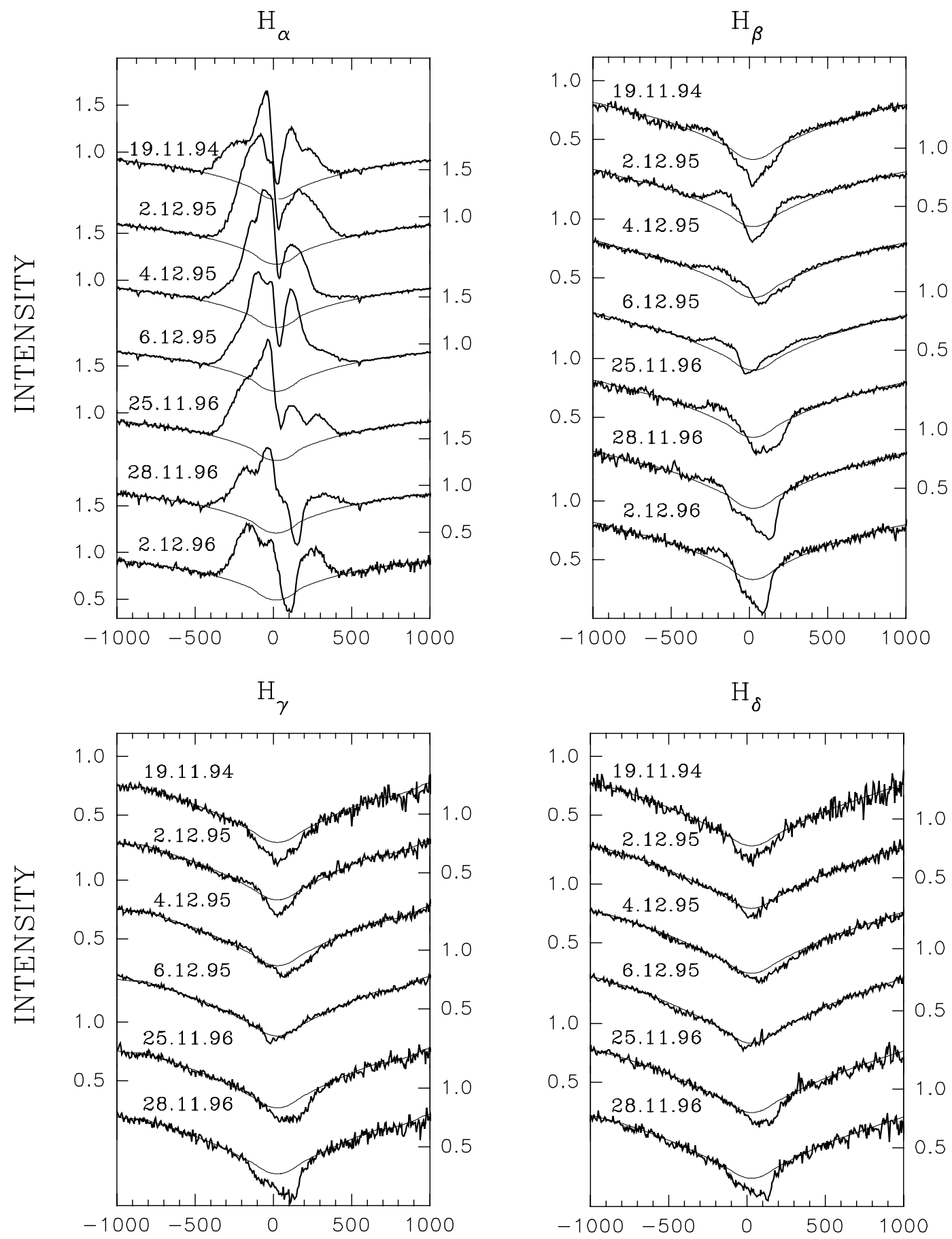

$\mathrm{V}_{\mathrm{r}}(\mathrm{km} / \mathrm{s})$

Fig. 1. a) Selected lines of UX Ori. Each spectrum has been normalized to the continuum and shifted vertically for easier display. Each curve is labelled with the date of the observation. The thin lines show the synthetic photospheric components.

lines are identified, and one can clearly see the photospheric spectrum of the star, as well as a large number of lines of CS origin.

As a general comment, we note that the spectra of these stars contain numerous absorption lines of neutral and ionized metals, as well as hydrogen lines, most prominently those of the Balmer series. Most of these lines are typical for normal stars of similar spectral types, but some, such as He I 5876, Na I D or the O I 7774 triplet, are not observed with the same intensity in normal A stars. We detect in the spectra numerous absorption lines of circumstellar origin, so-called shell or CS components. 


\section{UX Ori}

Ca II 3933

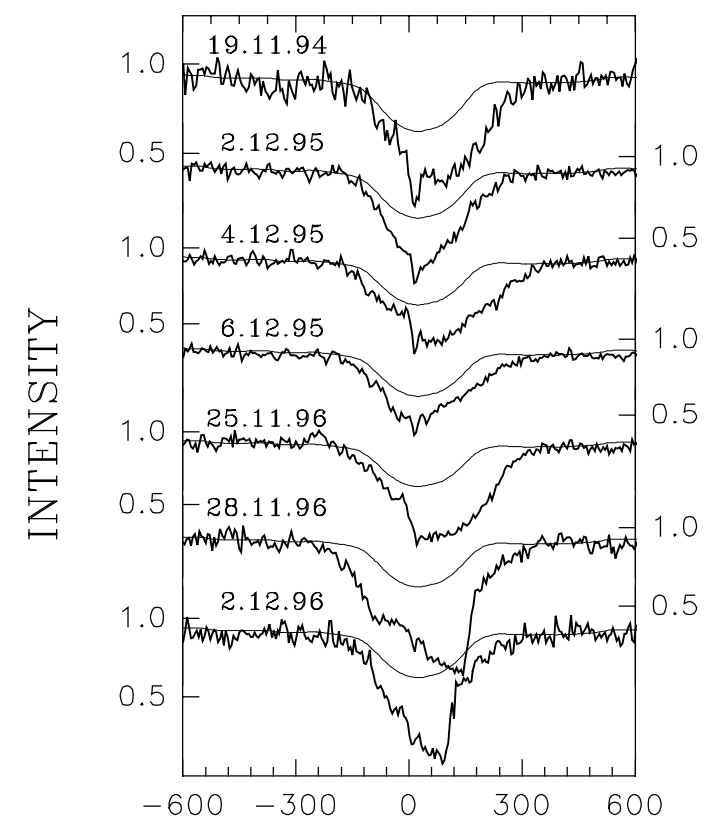

He I 5876

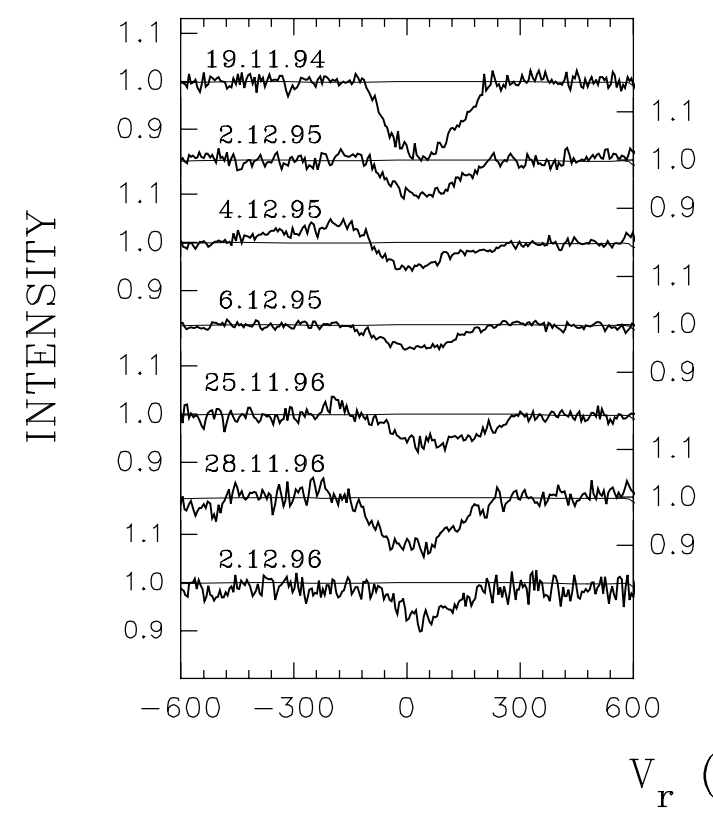

Fig. 1. b) Selected lines of UX Ori.

The presence of these components has to be taken into account in the analysis of the spectra and in estimating the stellar parameters (see Sect. 5).

\subsection{The $H_{\alpha}$ line}

In all the stars the $\mathrm{H} \alpha$ line shows the two-component profile that is typical of UX Ori-type stars (Grinin \& Rostopchina 1996). H $\alpha$ has broad, almost symmetric
Ca II 8662

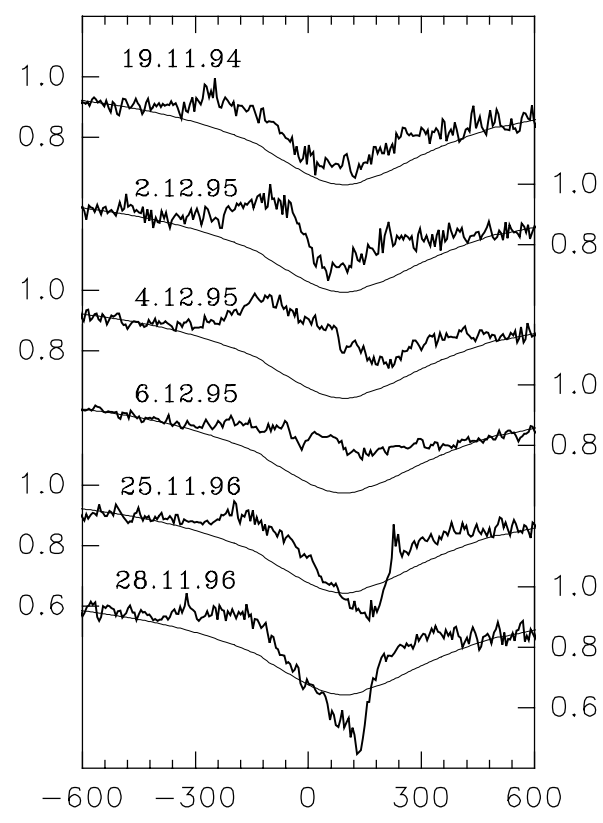

O I 77773

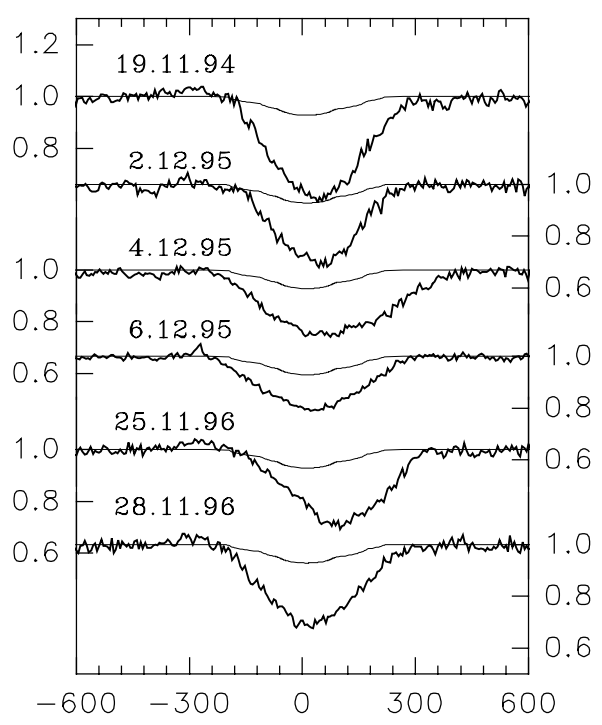

$(\mathrm{km} / \mathrm{s})$ wings in emission extending up to $\pm 400 \mathrm{~km} \mathrm{~s}^{-1}$. The typical radial velocities at half intensity are $\pm 200-300 \mathrm{~km} \mathrm{~s}^{-1}$.

The relative intensity of the two emission peaks varies from star to star and, for the same star, with time. It seems, however, that in spite of much time variation there is for each star a general pattern that characterizes the $\mathrm{H} \alpha$ profile. Namely, in RR Tau the red and blue peaks tend to have similar intensity, in UX Ori and CQ Tau the blue peak is systematically stronger than the red one (inverse 
UX Ori

$\mathrm{Si}$ II $6347 / 6371$

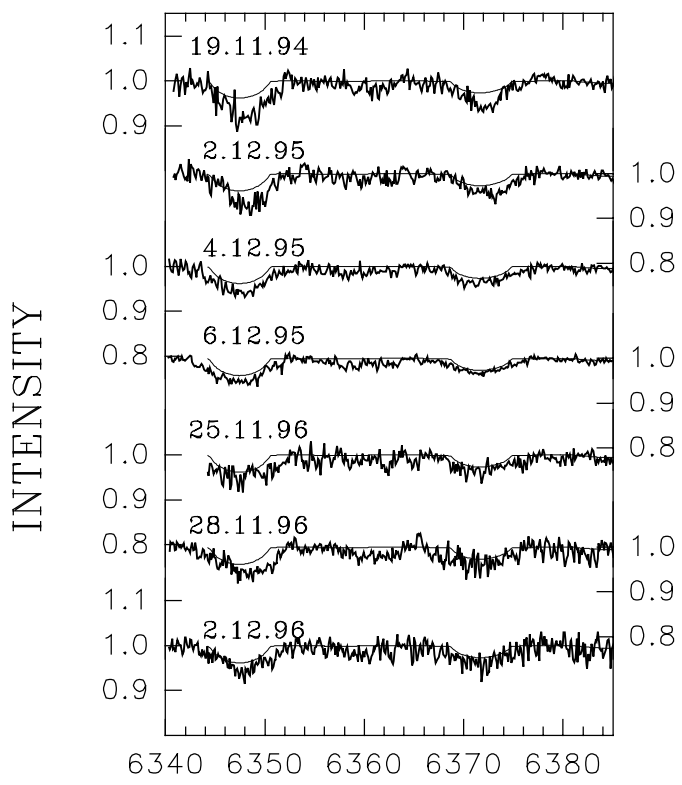

Fe II 5169, Mg I 5173

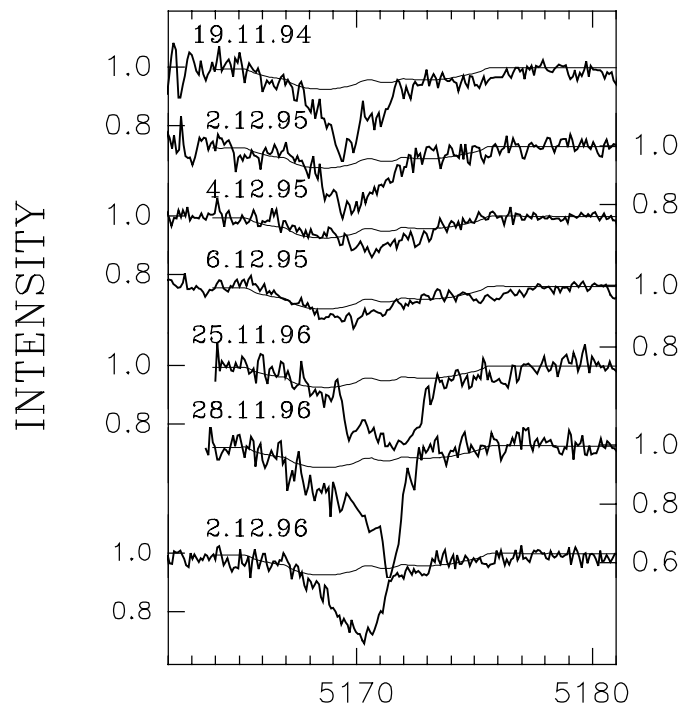

$\mathrm{Na}$ I $5889 / 5895$

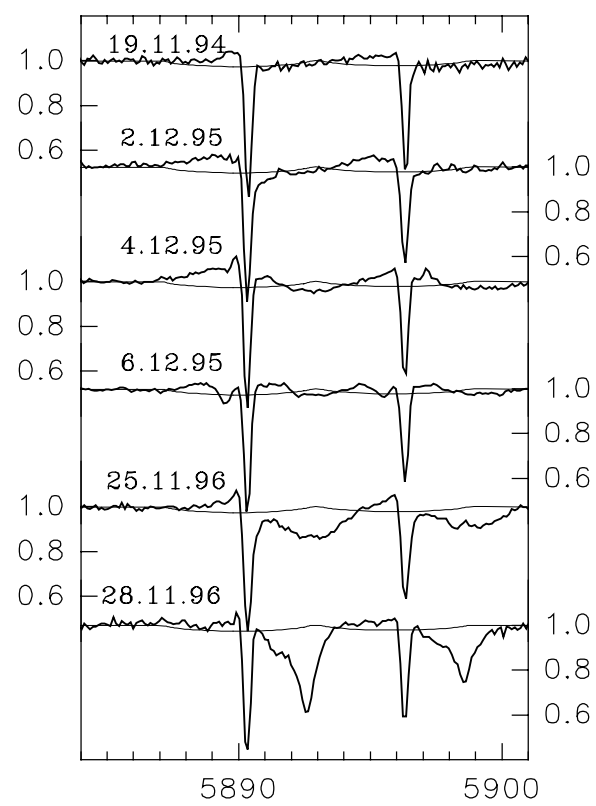

Ti II 4572, Fe II 4584

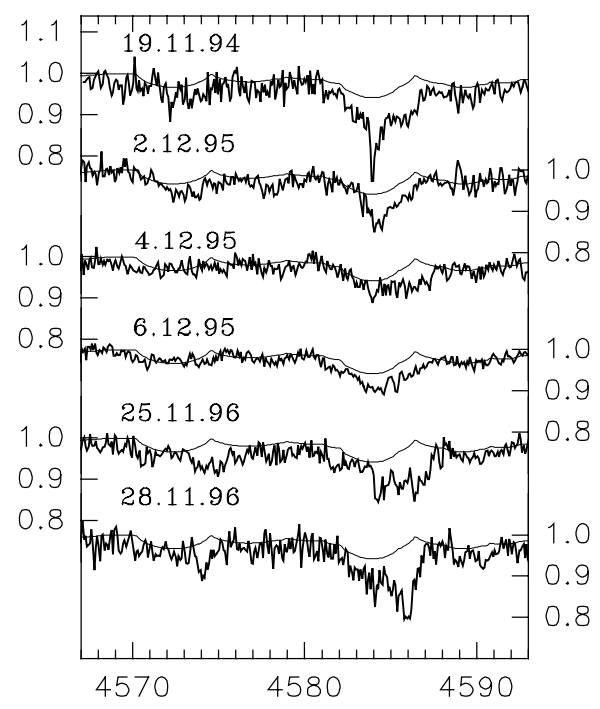

$\lambda(\mathrm{A})$

Fig. 1. c) Selected lines of UX Ori. Note that when more than one line is shown in a panel the horizontal scale is in wavelengths.

P Cygni type III profile), while in WW Vul the red peak is stronger than the blue one (P Cygni type III profile). This behaviour is confirmed by earlier observations (see, for example, for UX Ori Kolotilov 1977, Grinin et al. 1994, de Winter 1996; for WW Vul Reipurth et al. 1996; for CQ Tau Kozlova et al. 2000).

According to the interpretation given in Tambovtseva et al. (1999), these kinds of profiles expected if $\mathrm{H} \alpha$ forms in an accretion disk seen almost edge-on. The relative intensity of the blue and red peaks is determined by the combination of rotation and infall or outflow motions. If so, the symmetric profile of $\mathrm{H} \alpha$ in $\mathrm{RR}$ Tau and BF Ori suggests a situation where rotation dominates, the inverse P Cygni III profile of UX Ori and CQ Tau suggest a situation where infall motions are important and the P Cygni III profile of WW Vul a situation where outflow motions dominate the $\mathrm{H} \alpha$ formation region. Note, however, that at times the spectrum of WW Vul shows in some 


\section{UX Ori}
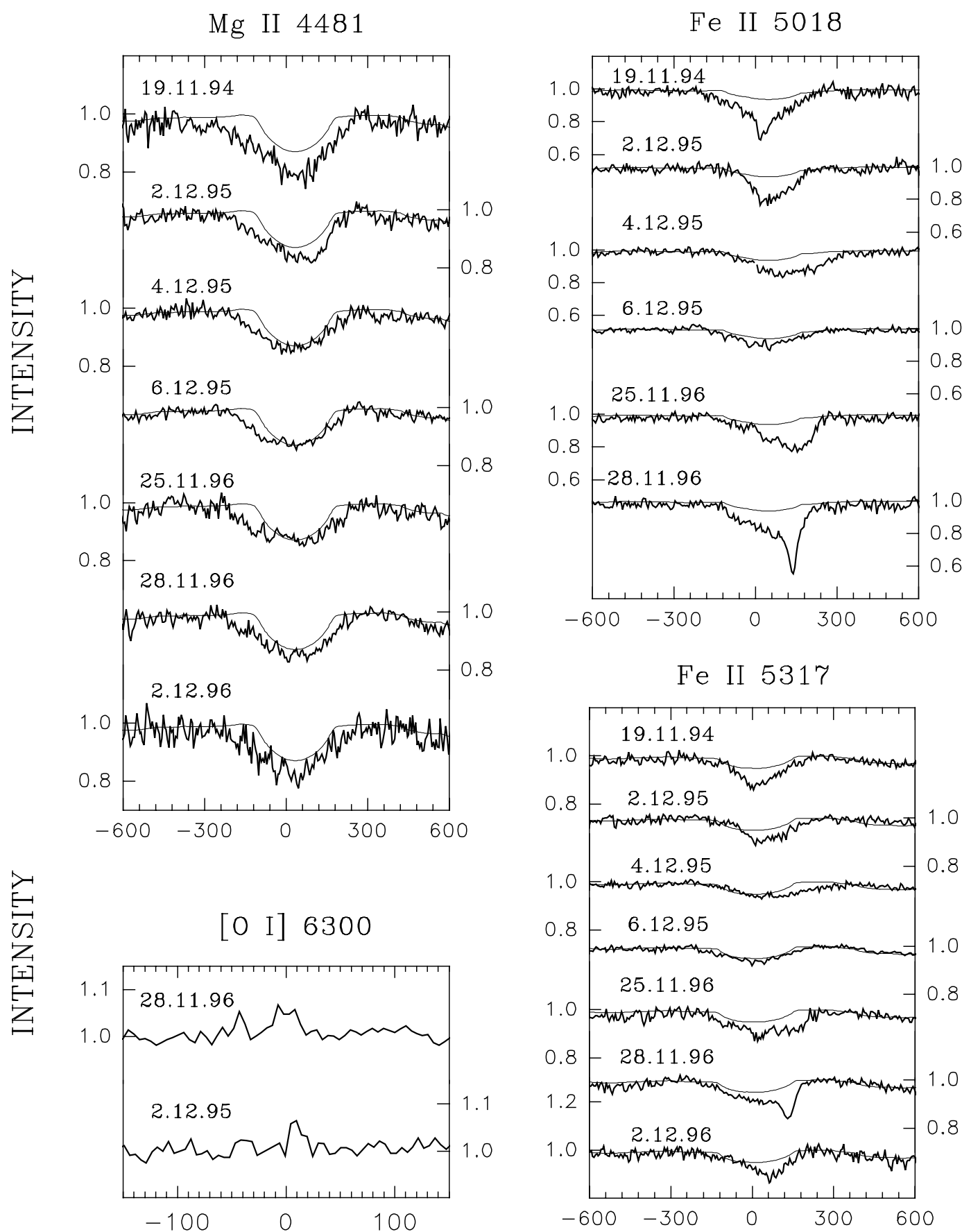

Fe II 5317

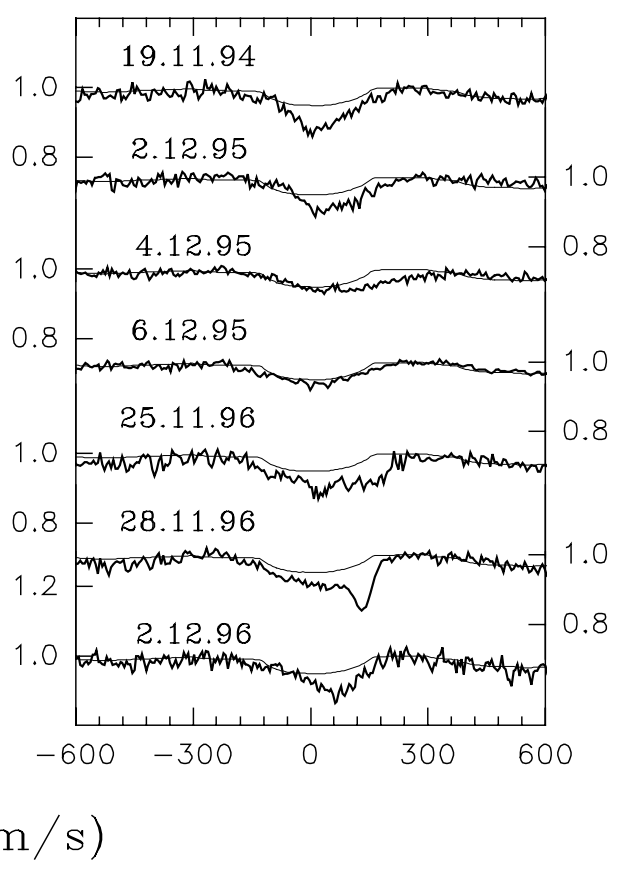

Fig. 1. d) Selected lines of UX Ori.

lines, such as He I 5876 and D Na I, red-shifted absorption components at high velocity, which are clear signatures of accreting gas (Fig. 5b and Grinin et al. 1996).

The equivalent width of $\mathrm{H} \alpha$ is given in Table 2. It has been computed as the emission in excess of the photospheric one, estimated from the synthetic spectra discussed in the following. With the exception of RR Tau, the values of $\mathrm{EW}(\mathrm{H} \alpha)$ are quite weak in comparison with other HAe stars. For example, among the 15 HAe stars listed by Bhöm and Catala (1995) only 7 have $W(\mathrm{H} \alpha)<$ $20 \AA$, and in the sample of 22 studied by Corcoran and Ray (1997) only 4. This last result is particularly significant because they did not subtract the photospheric spectrum, so that their values of $W(\mathrm{H} \alpha)$ are in fact lower limits. 


\section{UX Ori}

Fe II 5197

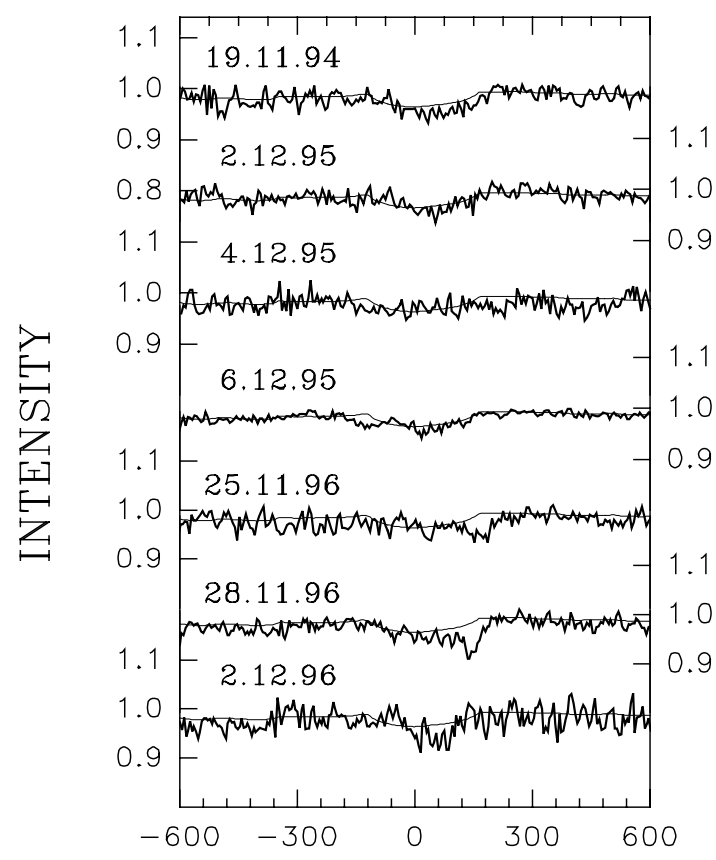

Mg I 5183

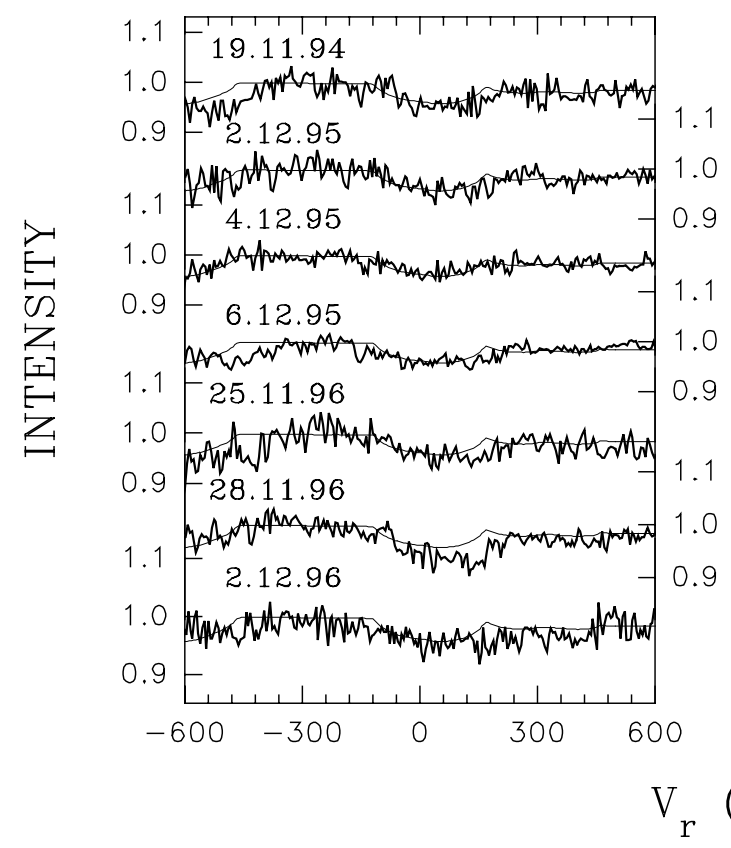

Fig. 1. e) Selected lines of UX Ori.

In $\mathrm{AB}$ Aur, one of the best studied HAe star with a disk seen close to face-on (Grady et al. 1999), $W(\mathrm{H} \alpha) \sim 40 \AA$ (Böhm \& Catala 1993; Pogodin 1992). The non-LTE models by Tambovtseva et al. (1999), who computed the $\mathrm{H} \alpha$ emission due to a circumstellar disk, show that this difference can be caused at least partially by a different disk orientation (more edge-on in UXORs, more face-on in AB Aur).
$\mathrm{Pa} 14$

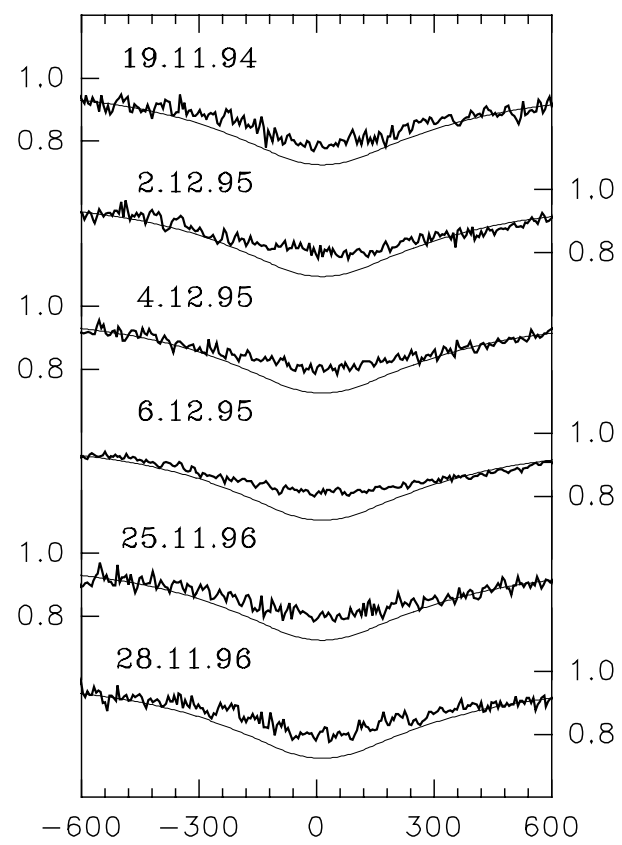

Ti II 4572

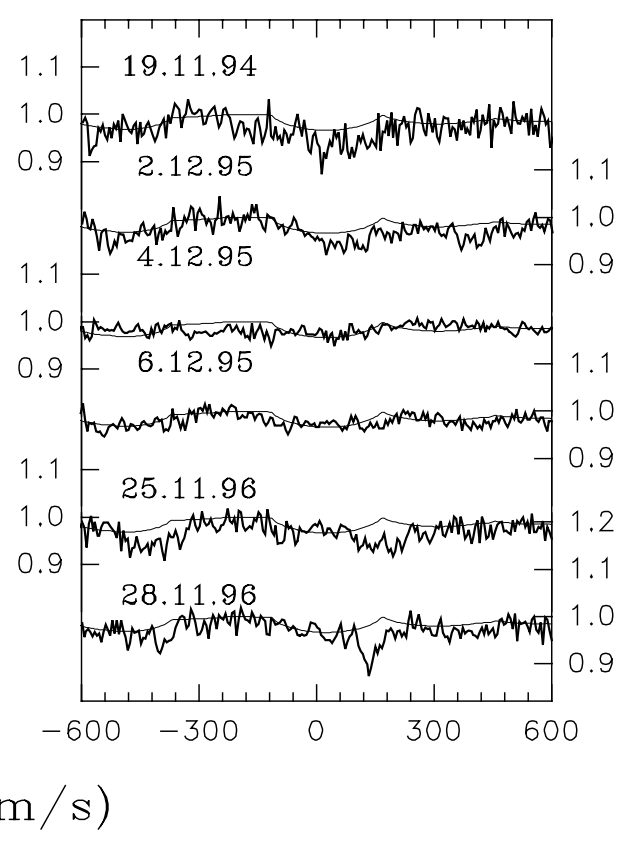

\subsection{HeI 5876 and OI 7774 lines}

These lines are seen in all spectra, and they are clearly of CS origin, since they are absent (He I 5876) or weak (O I 7774) in the photospheric spectra. They appear as broad (up to $\pm 200-300 \mathrm{~km} \mathrm{~s}^{-1}$ ) and highly variable absorption components, generally redshifted by less than $100 \mathrm{~km} \mathrm{~s}^{-1}$. In some spectra one sees weak blue-shifted 
Table 2. Log of the observations.

\begin{tabular}{|c|c|c|c|c|c|c|c|}
\hline \multirow[t]{2}{*}{ Name } & \multirow[t]{2}{*}{ Date } & \multirow{2}{*}{\multicolumn{2}{|c|}{$\begin{array}{r}V \\
\operatorname{mag}\end{array}$}} & \multirow[t]{2}{*}{$T$} & \multirow{2}{*}{$\begin{array}{l}\text { Exp } \\
\min \\
\end{array}$} & \multirow[t]{2}{*}{$S / N$} & \multirow{2}{*}{$\begin{array}{c}W(\mathrm{H} \alpha) \\
\AA\end{array}$} \\
\hline & & & & & & & \\
\hline \multirow[t]{8}{*}{ UX Ori } & 19.11 .94 & 9.70 & $:$ & 1 & 30 & 115 & 7.6 \\
\hline & 02.12 .95 & 9.74 & : & 1 & 45 & 140 & 10.6 \\
\hline & 04.12 .95 & 9.68 & & 1 & 45 & 170 & 10.5 \\
\hline & 06.12 .95 & 9.78 & & 1 & 90 & 230 & 10.1 \\
\hline & 25.11 .96 & 10.08 & & 1 & 60 & 60 & 8.4 \\
\hline & 28.11 .96 & 9.95 & & 1 & 20 & 90 & 5.5 \\
\hline & 28.11 .96 & 9.95 & & 2 & 25 & 95 & 5.5 \\
\hline & 02.12 .96 & 9.86 & & 2 & 40 & 95 & 5.2 \\
\hline \multirow{7}{*}{ CQ Tau } & 03.12 .95 & 9.80 & : & 1 & 60 & 120 & 8.4 \\
\hline & 05.12 .95 & 10.10 & & 1 & 60 & 170 & 5.1 \\
\hline & 21.11 .96 & 10.50 & & 1 & 60 & 120 & 12.0 \\
\hline & 26.11 .96 & 10.48 & & 1 & 60 & 130 & 8.6 \\
\hline & 29.11 .96 & 10.33 & & 1 & 30 & 100 & 7.5 \\
\hline & 29.11 .96 & 10.33 & & 2 & 40 & 80 & 7.6 \\
\hline & 02.12 .96 & 10.29 & & 2 & 40 & 50 & 6.1 \\
\hline \multirow[t]{5}{*}{ BF Ori } & 01.12 .95 & 10.83 & & 1 & 90 & 120 & 12.9 \\
\hline & 04.12 .95 & 10.94 & & 1 & 45 & 100 & 14.3 \\
\hline & 25.11 .96 & 9.93 & & 1 & 53 & 130 & 13.5 \\
\hline & 28.11 .96 & 9.97 & & 1 & 20 & 130 & 15.5 \\
\hline & 28.11 .96 & 9.97 & & 2 & 25 & 87 & 15.5 \\
\hline \multirow[t]{5}{*}{ RR Tau } & 03.12 .95 & 10.86 & : & 1 & 60 & 120 & 30.0 \\
\hline & 05.12 .95 & 11.03 & & 1 & 60 & 130 & 29.5 \\
\hline & 24.11 .96 & 12.35 & $:$ & 1 & 85 & 90 & 56.5 \\
\hline & 27.11 .96 & 10.86 & & 1 & 90 & 105 & 25.8 \\
\hline & 01.12 .96 & 10.89 & & 2 & 90 & 110 & 31.3 \\
\hline \multirow[t]{4}{*}{ WW Vul } & 20.11 .96 & 11.22 & $:$ & 1 & 45 & 90 & 20.7 \\
\hline & 25.11 .96 & 10.83 & & 2 & 90 & 100 & 21.5 \\
\hline & 29.11 .96 & 10.94 & & 2 & 80 & 90 & 21.0 \\
\hline & 01.12 .96 & 10.94 & & 2 & 56 & 50 & 23.9 \\
\hline
\end{tabular}

emission. In a few spectra there is indication of blueshifted absorption at low velocity (e.g., BF Ori, 4.10.95, 25.11.96) The absorption lines are broadened by differential motions of emitting gas (combination of infall and rotation) and formed in the nearest vicinity of the stars (see Tambovtseva et al. 1999 for a study of the He I 5876 line). Strong night-to-night variations of their profiles indicate strong fluctuations of the temperature and density along the line of sight in this region of the CS gas envelope.

\subsection{Sodium resonance lines}

The sodium D Na I resonance doublet is one of the most interesting spectral features of the UXORs spectra. Due to the low ionization energy $(5.17 \mathrm{eV})$ the sodium atoms are fully ionized in the atmospheres of A stars (see synthetic the spectra in Fig. 6) and in their immediate surroundings. Nevertheless the observations show that
RR Tau

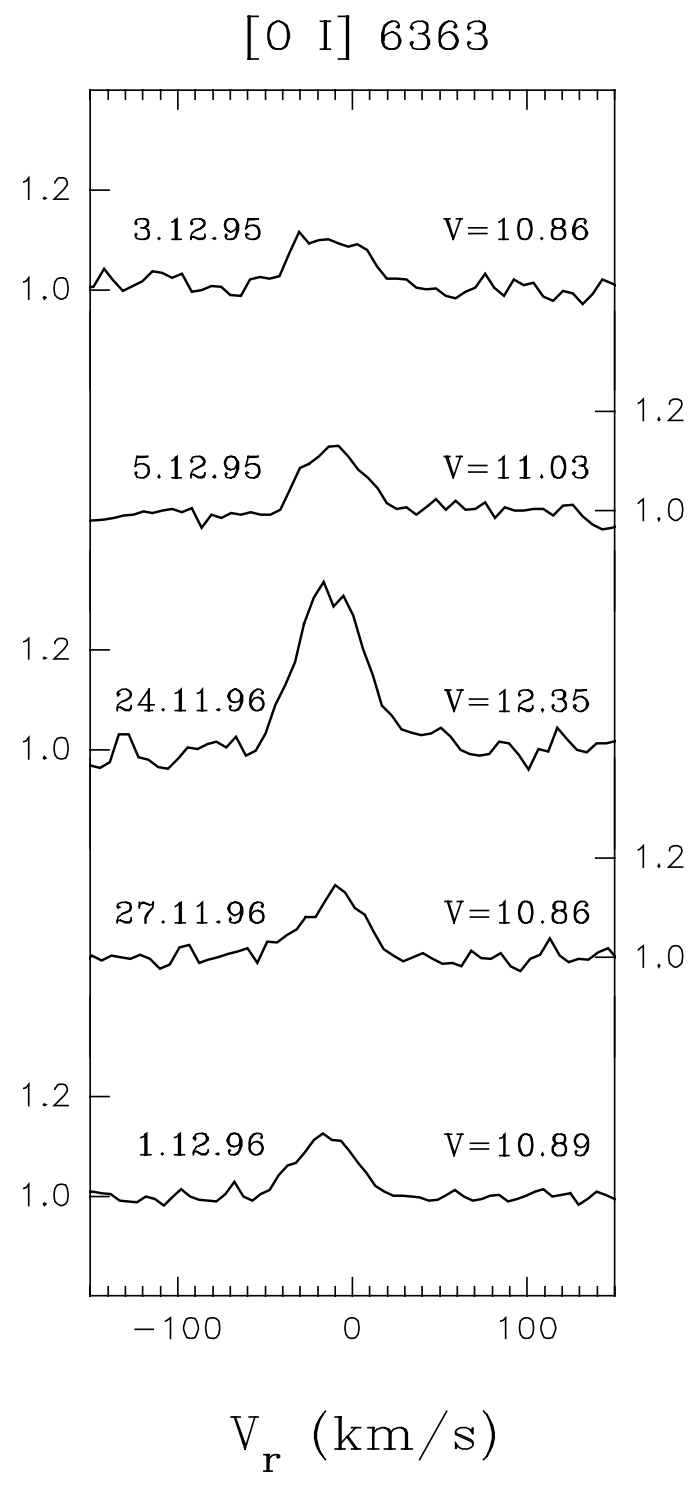

Fig. 4. d) [OI] $6363 \AA$ line at various epochs in RR Tau. The corresponding $V$ magnitude is given for each night.

variable CS components are present in the spectra of UXORs (Grinin et al. 1994, 1996; Grady et al. 1996; de Winter et al. 1999; Kozlova et al. 2000). In most cases one sees red-shifted absorption components with radial velocities up to $300 \mathrm{~km} \mathrm{~s}^{-1}$. These components can appear and disappear on time scale of days and even less without any visible brightness change of the stars. Sometimes the profiles show instead blue-shifted components indicating matter outflow. Blue-shifted absorption is frequently seen in the spectrum of WW Vul (Fig. 5c). In all stars, at times blue and red-shifted components are seen simultaneously (BF Ori, 1.12.95, Fig. 3c; RR Tau, 1.12.96, Fig. 4c). In all 
these cases the radial velocity of the blue-shifted components does not exceed velocities of roughly $100 \mathrm{~km} \mathrm{~s}^{-1}$.

The variable CS components of the D NaI lines are blended with narrow interstellar (IS) lines. According to Finkenzeller \& Jankovics (1984) the radial velocity of the IS lines coincides within a few kilometers per second with the radial velocity of the HAe star, and we have used it to estimate the values of the heliocentric radial velocity $V_{\mathrm{H}}$ given in Table 4.

The sodium resonance lines provide the clearest evidence that the gas motions in UXORs are very complex, with infall and outflow occurring in the same objects, sometime simultaneously.

\subsection{Pashen 14 line}

The CS component of this hydrogen line is usually weak in the spectra of UXORs (see Fig. 1e). Nevertheless, it is important for diagnostics since the line is usually optically thin, and the upper level of this transition is known with high accuracy in LTE. Therefore the emission of the line (after subtraction of the photospheric component) is proportional to the emission measure of the emitting region (Tambovtseva et al. 2001).

\section{Spectral variability of UXORs}

In the case of UXORs we deal with two different sources of spectral variability. One of them is typical of many stars with CS gas envelopes and is connected with variations of temperature and density in the emitting region and along the line of sight. The observations show that such variations can be quite strong and lead to strong variability of the spectral lines. In particular, we want to call attention to the He I 5876 line (Figs. 1b-5b), which has the highest excitation energy (about $21 \mathrm{eV}$ ) of its lower level of all the lines in our spectra and is, therefore, the most sensitive to temperature fluctuations.

The other mechanism of spectral variability is quite specific for UX Ori-type stars. It is connected to the coronagraphic effect produced by the screens of dust that sporadically obscure the star and cause the deep minima that characterize UXORs. For convenience, in the following we will call such screens "clumps", although their geometry and origin are at present uncertain. When it intersects the line-of-sight and screens the star from the observer, a CS clump screens at the same time the central part of the gas envelope where the CS lines form. The outer part of the CS envelope, on the other hand, is not screened. This causes an increase of the strength of the emission component with respect to the stellar continuum, whose degree depends on the extension of the line emitting region with respect to the extension of the obscuring clump. We expect that this effect will be maximum for strong emission lines, such as $\mathrm{H} \alpha$ and minimum for the weaker lines that are seen mostly as absorption against the stellar photosphere.

The high resolution spectra of UX Ori around $\mathrm{H} \alpha$ obtained at the moment of a very deep minimum in August
$1992(\Delta V=2.5 \mathrm{mag}$, Grinin et al. 1994) have shown that not only the equivalent width of $\mathrm{H} \alpha$ changes when the star fades but also the line profile, which turns from its usual double-peaked shape to single peak. Such changes are the consequence of the coronagraphic effect caused by CS clumps: when screening the star from the observer, they screen also that part of the CS gas envelope in front of the star which is responsible for the absorption component of the line profile. As a result the central absorption decreases and can disappear completely (see Grinin \& Tambovtseva 1995).

We have among our spectra of RR Tau the most convincing case of a minimum, when the star fades by about $1.6 \mathrm{mag}$ (24.11.96). One can see the strong relative increase of $\mathrm{H} \alpha$ whose equivalengt width increases by almost a factor of two (Table 2). Similar changes have been found by Kolotilov (1977) on the basis of low resolution spectra and by Herbst et al. (1983) from narrow-band photometry. However, the 24.11.96 minimum of RR Tau was not a very deep one for this star (see i.e. Rostopchina et al. 1997), and the transformation of the $\mathrm{H} \alpha$ line profile from double to single peaked, observed in the 1992 minimum of UX Ori, was not detected.

It is interesting to note that a strong increase relative to the stellar continuum is seen in RR Tau also in the forbidden line [O I] 6363, whose equivalent width increased in the 24.11.96 night by about a factor of three, from 0.11 to $0.33 \AA$, as the star faded by a similar amount (Fig. $4 \mathrm{~d})^{2}$. A similar increase of the equivalent width of the [O I] 6363 line was also observed in CQ Tau in 2.12.96 (Fig. 2d), when the star, however, was close to its maximum brightness.

The behaviour of CS lines during the photometric minima of UXORs supports the interpretation of the observed anti-correlation between linear polarization of UXORs and their brightness (Grinin et al. 1991) and gives us additional and independent evidence that the deep minima of UXORs are caused by variable obscuration of the star by CS dust clouds.

\section{Stellar parameters}

For all the five stars we have computed a typical spectrum by averaging all the spectra obtained when the star was in its bright state. These average spectra have then been compared to synthetic spectra calculated from the Kurucz (1993) models of stellar atmospheres (for solar chemical composition) and the list of spectral lines from the data base VALD (Kupka et al. 1998). We have varied effective temperature and gravity and convolved the result with different values of the projected rotation velocity $v \sin i$ until a good fit to the most stable photospheric features was achieved. The values of $V_{\mathrm{H}}$ given in Table 3 have been adopted for each star. We have relied in particular on the photospheric wings of the Balmer lines, and

\footnotetext{
${ }^{2}$ A similar effect was recently observed by B. Rodgers in a deeper minimum of RR Tau (Rodgers et al. 2001).
} 

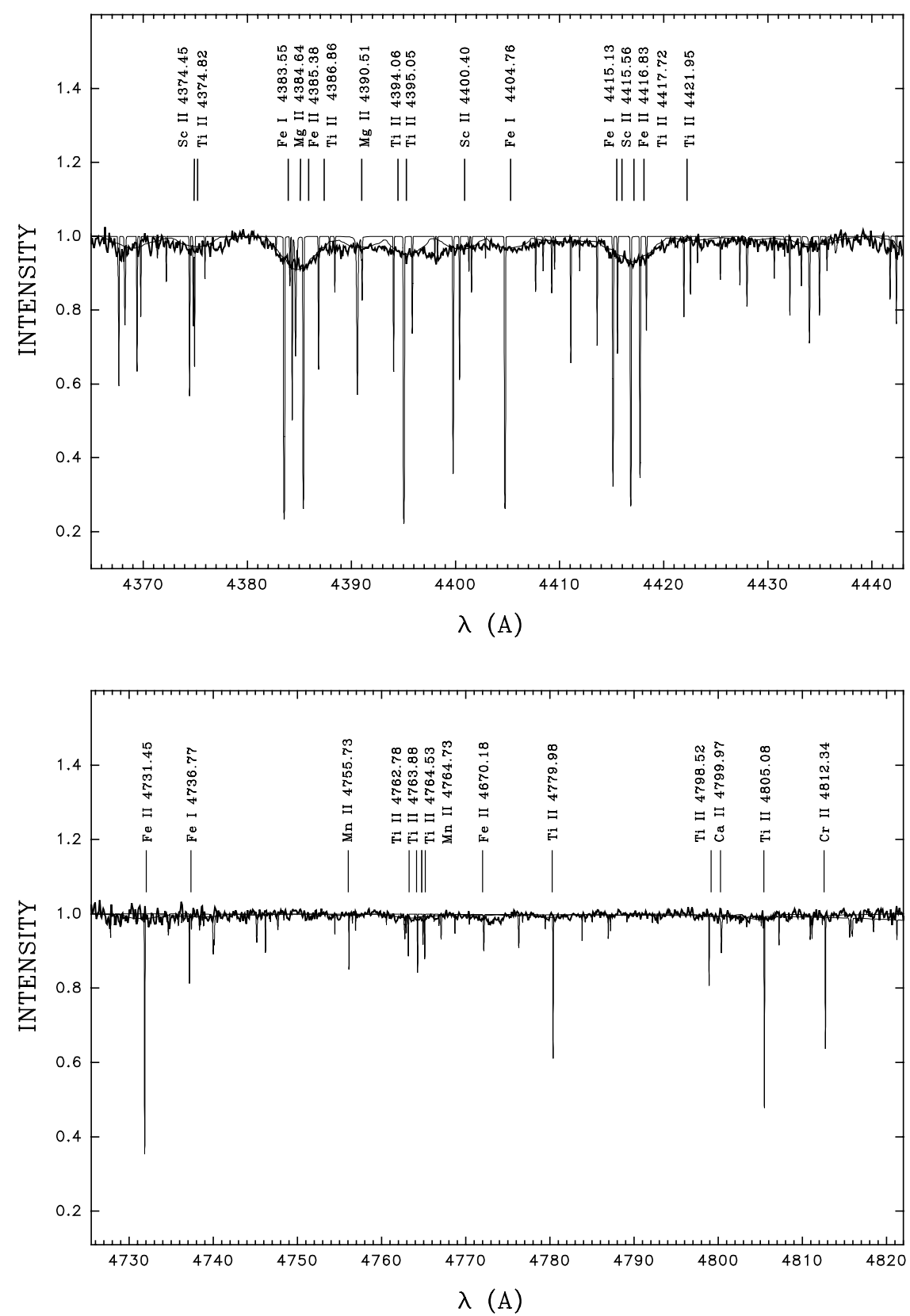

Fig. 6. Complete spectrum of UX Ori (selected regions). The spectrum is the average of the three 1996 nights when the star was at maximum brightness. The gray line shows the synthetic spectrum for $T_{\text {eff }}=9500 \mathrm{~K}$ and $\log g=4.0$. The black thin line shows the same spectrum after convolution with a projected rotation velocity $v \sin i=140 \mathrm{~km} \mathrm{~s}^{-1}$. The strongest lines are identified. The complete spectrum is available electronically.

supplemented our data with the low resolution spectra by Kozlova et al. (1995). All calculations were made with the codes SYNT and ROTATE by Piskunov (1992). The best values of $T_{\text {eff }}, \log g$ and $v \sin i$ are shown in Table 3 . Note that our values of $\log g$ are systematically higher than those determined by Kovalchuk \& Pugach (1997), based on lower resolution spectra.

The full average spectrum of UX Ori is shown in Fig. 6 . We show in the same figures the best synthetic spectrum before and after convolution with the projected rotation velocity. One can see the good agreement between observed and synthetic spectra in the Balmer wings as well as in the numerous weak metallic lines. On the contrary, strong metallic lines like Fe II or Mg II tend to be deeper then predicted. Inspection of the individual spectra shows that their shape changes with time, indicating a strong contribution of a CS component. As we have already discussed, some spectral lines like He I 5876 have a purely CS origin and originate probably in the inner part of an accretion disk (Tambovtseva et al. 1999).

We did not find any signature of veiling of the photospheric lines by a non-stellar continuum emission. 
Table 3. New values of the stellar parameters.

\begin{tabular}{lcccc}
\hline \hline Name & $\begin{array}{c}T_{\text {eff }} \\
(\mathrm{K})\end{array}$ & $\log g$ & $\begin{array}{c}V_{\mathrm{H}} \\
\left(\mathrm{km} \mathrm{s}^{-1}\right)\end{array}$ & $\begin{array}{c}v \sin i \\
\left(\mathrm{~km} \mathrm{~s}^{-1}\right)\end{array}$ \\
\hline UX Ori & 9500 & 4.0 & +18 & 140 \\
CQ Tau & 7000 & 3.5 & +20 & 90 \\
BF Ori & 8750 & 4.0 & +18 & 40 \\
RR Tau & 9750 & 3.5 & +11 & 140 \\
WW Vul & 8500 & 3.5 & -12 & 150 \\
\hline \hline
\end{tabular}

This is also typical of other non-embedded HAEBE stars (Corcoran \& Ray 1994; Ghandour et al. 1994).

\section{Discussion}

The optical spectra of these UXORs, which are among the most active in the group, can be described in important detail by the synthetic spectra of normal A stars. This is the case, for example, for the broad wings of the Balmer lines as well as for numerous weak metallic lines. The strong metallic lines are usually blended with shell components. The comparison with the high resolution spectrum of the photometrically non-active Herbig Ae star AB Aur (Böhm \& Catala 1993) shows that the shell components are more numerous in the spectra of UXORs. We think that this is a consequence of the edge-on orientation of the disks of UXORs relatively to the observer, and we suggest that these lines can be used to further study the structure of the disk in the vicinity of the star. It should be noted that the presence of many CS metal lines causes serious problems in the determination of the stellar parameters. A preliminary study of the time stability of each feature is necessary to define the "true" photospheric spectrum one wants to fit.

In some stars the spectral signatures of accretion and outflow can be observed simultaneously. For example in the 20.11.96 spectrum of WW Vul (Fig. 5a) $\mathrm{H} \alpha$ has a direct $\mathrm{P}$ Cygni type III profile, while $\mathrm{H} \beta$ and $\mathrm{H} \gamma$ have a characteristic type III inverse $\mathrm{P}$ Cygni profile with a weaker blue-shifted emission component. The same combination of the line profiles was observed on the night 1.12.96. Simultaneous infall and outflow motions are commonly observed in UXORs, as well as in many $\mathrm{T}$ Tauri stars (see, for example, Edwards et al. 1984; Johns \& Basri 1995; Petrov et al. 1996), where they are both interpreted as phenomena associated with disk accretion. The analysis of the spectra of UXORs will show if the same interpretation can be applied to higher mass stars as well.

\subsection{The gas accretion rate}

The observations of the Balmer lines shown in this paper, together with the Pashen 14 and Brackett gamma lines, were used recently by Tambovtseva et al. (2001) to estimate the gas accretion rate $\dot{M}_{\mathrm{a}}$. These authors have assumed that the lines form in the inner region of an accretion disk illuminated by the central star and performed a non-LTE analysis of the physical conditions of the gas. They derive a typical value of the gas accretion rate for UX Ori of about $(2-5) \times 10^{-9} M_{\odot}$ per year. This is significantly less then the value $\dot{M}_{\mathrm{a}}=10^{-6} M_{\odot}$ per year suggested recently in the papers by Herbst \& Shevchenko (1999) and Bertout (2000).

Small accretion rates agree with the absence of veiling in the spectra of UXORs (see Sect. 5). Also, the low $\dot{M}_{\mathrm{a}}$ in UXORs are in agreement with the mass of their CS disks $M_{\text {csd }}$ based on observations at millimeter wavelenghts (Natta et al. 1997). For example, in the case of UX Ori $M_{\text {csd }}=0.04 M_{\odot}$. The age of this star is about $3 \times 10^{6}$ yrs (Natta et al. 1997; Rostopchina 1999) and hence the mean value of the mass accretion rate is about $10^{-8} M_{\odot}$ per year, i.e., only a few times greater then the value derived from the hydrogen lines.

\subsection{Origin of the redshifted absorption components in the sodium $\mathrm{D} \mathrm{Nal}$ lines}

Variable red-shifted absorption components in the sodium D Na I resonance lines are frequently observed in the spectra of UXORs and their origin is a matter of debate (see Grady et al. 2000 and reference therein). Their properties are very similar to those observed in the spectra of $\beta$ Pictoris in the Ca II resonance lines (Ferlet et al. 1987). In the case of UXORs the highest radial velocity of the infalling gas reaches $300 \mathrm{~km} \mathrm{~s}^{-1}$. The problem is connected with the fact that sodium is quickly ionized in the vicinity of HAe stars, where a sizeable amount of neutral atoms can be obtained in two cases: 1) when the clump has a strong excess of metals relative to the solar abundance, or 2) when it has normal chemical composition, but high density (Sorelli et al. 1996).

The accretion event which has been observed in the spectrum of UX Ori in the night of 28.11.96 turned out to be important for discriminating between these two possibilities. It was so strong that red-shifted absorption components were clearly seen not only in the resonance lines, such as $\mathrm{Na} \mathrm{D}$, but also in many other metallic lines and, of particular importance, in all the Balmer lines visible in the spectrum (Fig. 1a). Non-LTE modeling of the lines optical depth has shown that the infalling gas in that event could not be heavily hydrogen-depleted, as would be expected if it was produced by the evaporation of a solid body of chemical composition similar to solar system comets, but had a chemical composition approximately solar (Natta et al. 2000b). Evaporating comet-like or planetesimal bodies cannot be the main source of matter accreting onto UX Ori-type stars. Thus, these stars can be consider only as remote, rather than immediate progenitors of $\beta \mathrm{Pic}$, as indicated also by the large difference between their IR excesses: in the case of UXORs they are similar to those observed in the classical HAe stars (Grinin et al. 1991). 


\section{Summary}

We have presented in this paper the high resolution spectra of five UXORs (UX Ori, CQ Tau, BF Ori, RR Tau, WW Vul), which cover the entire visual range, from 3900 to $8700 \AA$. There are between 4 and 7 spectra per star, obtained over a time interval of two years. Simultaneous or quasi-simultaneous photometric observations were obtained at the Crimean Astrophysical Observatory. The spectra are too sparse to form true time sequences; however, they provide an extremely useful database for studying the photospheric spectrum of the stars and determining stellar parameters such as the effective temperature, stellar gravity and rotational velocity, as well as for investigating the circumstellar environment and its variability. The complete, reduced and normalized spectra are available in electronic form. Here we have shown for each star a selection of the most interesting lines, and the full spectrum of UX Ori computed by averaging the spectra obtained when the stars were at maximum light. We show also the synthetic spectrum and provide an identification of most of the lines.

The main features of the spectra have been discussed briefly, to indicate how they can be used to study the properties of UXORs. However, we want to stress that the wealth of information they contain has by no means been entirely exploited.

Among the points we have touched, we have shown that the spectra of UXORs contain many photospheric lines that can be described in good approximation by the synthetic spectra of normal A stars with $\log g=3.5-4$. This conclusion agrees with previous estimates of the evolutionary status of UXORs as HAe stars, based on the analysis of their IR excess (Grinin et al. 1991; Natta et al. 1997).

In addition to this normal photospheric spectrum, there is a large number of absorption components of circumstellar origin (shell-components) visible in the strong metallic lines. Most of them are highly variable and redshifted, indicating non-stationary accretion. This is specific of UXORs (also with respect to other, less variable HAEBE stars of similar spectral type) and is probably caused by the orientation of their CS disks, which in UXORs is almost edge-on for the observer. One object (WW Vul) shows simultaneous evidence of infall and outflow motions. Strong emission is seen only in the lower Balmer lines. When interpreted as forming in the inner regions of an accretion disk, they can be used to estimate the accretion rate, which turns out to be rather low (2$5 \times 10^{-9} M_{\odot} \mathrm{yr}^{-1}$ in UX Ori; Tambovtseva et al. 2001).

Most lines vary strongly with time. The variability is in many cases not related to the brightness variation of the star and is caused by perturbations of the gas density, electron temperature and the kinematic conditions in the CS environment. The strongest variations of such a type are observed in He I 5876 and O i 7774 lines. There is, however, an additional source of spectral variability, which is related to the brightness variations that characterize UXORs. It is connected to the coronagraphic effect produced by the dust clouds that sporadically obscure the star and part of the region where the CS line is formed. Spectral variability of such a kind is seen in the present database only in one RR Tau spectrum, when the star fades by about $1.6 \mathrm{mag}$, in both $\mathrm{H} \alpha$ and the [O I] $6364 \AA$ line.

We did not find any evidence of veiling of the photospheric lines by a non-stellar continuum, although we have not set an upper limit to it. This agrees with the low level of accretion activity of UXORs estimated from the nonLTE analysis of the hydrogen lines and with the absence of veiling in the spectra of non-embedded HAEBE stars in general. The recent idea of Herbst \& Shevchenko (1999) that accretion plays a dominant role in UXORs seems to have no observational support.

Acknowledgements. Many thanks to the referee, Carol Grady, for her help in organizing the material shown in this paper. V. P. Grinin thanks Franco Pacini for hospitality during his stay in the Arcetri Astrophysical Observatory where part of this work was done. This work was partly supported by the RFBR grant 99-02-18520.

\section{References}

Bertout, C. 2000, A\&A, 363, 984

Böhm, T., \& Catala, C. 1993, A\&ASS, 101, 629

Böhm, T., \& Catala, C. 1995, A\&A, 301, 155

Corcoran, M., \& Ray, T. 1994, PASPC, 62, 151

Corcoran, M., \& Ray, T. 1997, A\&A, 321, 189

Edwards, S., Hartigan, P., Ghandour, L., \& Andrulis, C. 1994, AJ, 108, 1056

Eiroa, C., Garzon, F., Miranda, L. F., \& EXPORT 2000, in Disks, Planetesimals and Planets, ed. F. Garzon, C. Eiroa, D. de Winter, \& T. J. Mahoney, PASPC, 219, 363

Ferlet, R., Vidal-Madjar, A., \& Hobbs, L. M. 1987, A\&A, 185, 267

Finkenzeller, U., \& Jankovics, I. 1984, A\&ASS, 57, 285

Finkenzeller, U., \& Mundt, R. 1984, A\&ASS, 55, 109

Ghandour, L., Strom, S., Edwards, S., \& Hillenbrand, L. 1994, PASPC, 62, 227

Graham, J. 1992, PASP, 104, 479

Grady, C. A., Pérez, M., Talavera, A., et al. 1996, A\&ASS, 120,157

Grady, C. A., Woodgate, B., Bruhweiler, F. C., et al. 1999, ApJ, 523, L151

Grady, C. A., Sitko, M. L., Russell, R. W., et al. 2000, in Protostars and Planets IV, ed. V. Mannings, et al. (The University of Arizona Press, Tucson), 613

Grinin, V. P. 1988, Sov. Astron. Lett. 14, 27

Grinin, V. P., Kiselev, N. N., Minikulov, N. Kh., \& Chernova, G. P. 1988, Pis'ma v Astron. Zn. 14, 514

Grinin, V. P. 1994, in The Nature and Evolutionary Status of Herbig Ae/Be stars, ed. P. S. Thé, M. R. Pérez, \& E. P. J. van den Heuvel, ASP Conf. Ser., 62, 63

Grinin, V. P., \& Rostopchina, A. N. 1996, Astron. Rep., 40, 171

Grinin, V. P., \& Tambovtseva, L. V. 1995, A\&A, 293, 396

Grinin, V. P., Kiselev, N. N., Minikulov, N. K., Chernova, G. P., \& Voshchinnikov, N. V. 1991, Ap. Sp. Sci, 186, 283 
Grinin, V. P., Thé, P. S., de Winter, D., et al. 1994, A\&A, 292, 165

Grinin, V. P., Kozlova, O. V., Thé, P. S., \& Rostopchina, A. N. 1996, A\&A, 309, 474

Hamann, F., \& Persson, S. E. 1992, ApJS, 82, 247

Herbig, G. H. 1960, ApJS, 4, 337

Herbst, W., Holtzman, J. A., \& Klasky, R. S. 1983, AJ, 88, 1648

Herbst, W., \& Shevchenko, V. S. 1999, AJ, 118, 1043

Ilyin, I. V. 2000, Ph.D. Thesis, University of Oulu

Ilyin, I. V., \& Duemmler, R. 1999, in Precise stellar radial velocities, IAU Colloq. 170, ed. J. B. Hearnshaw, \& C. D. Scarfe, PASPC, 185, 63

Johns, C. M., \& Basri, G. 1995, AJ, 449, 341

Kolotilov, E. A. 1977, Astrofizika, 13, 33

Kovalchuk, G. U., \& Pugach, A. F. 1997, A\&A, 325, 1077

Kozlova, O. V., Grinin, V. P., \& Rostopchina, A. N. 1995, AAT, 8, 249

Kozlova, O. V., Grinin, V. P., \& Rostopchina, A. N. 2000, Astron. Rep., 44, 36

Kupka, F., Piskunov, N. E., Ryabchikova, T. A., Stempels, T. A., \& Weiss, W. W. 1998, Vienne data base VALD

Kurucz, R. 1993, CD-ROM No. 13, Cambridge, Mass.: Smithsonian Astrophysical Observatory

Natta, A., \& Whitney, B. A. 2000, A\&A, 364, 633.

Natta, A., Grinin, V. P., Mannings, V., \& Ungerechts, H. 1997, ApJ, 491, 885

Natta, A., Grinin, V., \& Mannings, V. 2000a, in Protostars and Planets IV, ed. V. Mannings et al. (The University of Arizona Press, Tucson), 559

Natta, A., Grinin, V., \& Tambovtseva, L. 2000b, ApJ, 542, 421

Pérez, M. R., \& Grady, C. A. 1997, Space Sci. Rev., 82, 407
Petrov, P. P., Gullbring, E., Ilyin, I., et al. 1996, A\&A, 314, 821

Piirola, V. 1975, Ann. Acad. Sci. A, VI, 61

Piskunov, N. E. 1992, in Stellar Magnetizm, ed. Yu.V.Glagolevsky \& I. I. Romanjuk (St. Petersburg, Nauka), 92

Pogodin, M. A. 1992, Astron. Lett., 18, 1066

Reipurth, Bo, Pedrosa, A., \& Lago, M. T. V. T. 1996, A\&AS, 120, 229

Rodgers, B., Wooden, D., Grinin, V., et al. 2001, ApJ, in press

Rostopchina, A. N. 1999, Astron. Rep. 43, 113

Rostopchina, A. N., Grinin, V. P., Okazaki, A., et al. 1997, A\&A, 327, 145.

Sorelli, C., Grinin, V. P., \& Natta, A. 1996, A\&A, 309, 155

Tambovtseva, L. V., Grinin, V. P., \& Kozlova, O. V. 1999, Astrofizika, 42, 75

Tambovtseva, L. V., Grinin, V. P., Rodgers, B., \& Kozlova, O. V. 2001, Astron. Rep., 45, 445

Thé, P. S., de Winter, D., \& Pérez, M. R. 1994, A\&AS, 104, 315

Tuominen, I., Ilyin, I., \& Petrov, P. 1999, in Astrophysics with the NOT, ed. H. Karttunen, \& V. Piirola (Univ. Turku), 47

Wallace, L., Hinkle, K., \& Livingston, W., An atlas of spectrum of the solar photosphere from 13500 to $28000 \mathrm{sm}-1$, NSO Technical Report N 98-001

de Winter, D. 1996, Observational aspects of Herbig $\mathrm{Ae} / \mathrm{Be}$ stars and of candidate young A/B stars, Thesis, Amsterdam

de Winter, D., Grady, C., van den Ancker, M. E., et al. 1999, A\&A, 343, 137 